\title{
Computed tomography-derived pathological phenotypes in COPD
}

\author{
William MacNee
}

Affiliation: University of Edinburgh/MRC Centre for Inflammation Research, Queen's Medical Research Institute, Edinburgh, UK.

Correspondence: William MacNee, University of Edinburgh/MRC Centre for Inflammation Research, Queen's Medical Research Institute, 47 Little France Crescent, Edinburgh, EH16 4TJ, UK. E-mail: w.macneeded.ac.uk

0 @ERSpublications

There is a need to reach a consensus on the most appropriate method for quantifying emphysema and airways by CT http://ow.ly/YCKP300tQQi

Chronic obstructive pulmonary disease (COPD) is considered to be a complex, multicomponent, heterogeneous disease, the pathology, clinical and functional presentation of which vary greatly between individual patients despite similar degrees of airflow limitation [1]. COPD has therefore been considered to have distinct phenotypes that represent a single or combination of disease attributes that describe differences between individuals with COPD as they relate to clinically meaningful outcomes such as symptoms, exacerbations, rate of disease progression or death [2]. Importantly, these phenotypes of COPD may require different targeted treatment strategies [3].

From the early descriptions of COPD, the major pathological features of COPD (chronic bronchitis in the large airways, emphysema in the lung parenchyma and small-airway disease in airways less than $2-3 \mathrm{~mm}$ in diameter) have been considered as potential phenotypes of COPD. Since the first description, over 35 years ago, of the use of computed tomography (CT) lung density measurements to quantify the extent of emphysema [4], CT lung imaging has been used as a noninvasive method to evaluate changes in pulmonary structure [5] and thus to assess the pathological phenotype in COPD patients [6].

Emphysema was the first pathological phenotype to be assessed by CT imaging where the lung density value (in Hounsfield units (HU)) characterising the fifth percentile (and later the 15th percentile (Perc15/PD15)) of the frequency histogram of lung density values was shown to correlate with morphometric measurements of airspace size [7]. Other reports have used the percentage of low-attenuation lung voxels below a threshold value, commonly of $-950 \mathrm{HU}$ (\% low attenuation areas (\%LAA) or relative area -950 (RA-950)), and shown it to have the strongest pathological correlation with both macroscopic and microscopic emphysema $[8,9]$. It is now fairly well established that both measures are reliable and reproducible measures to quantify emphysema in cross-sectional studies [10]. However, the use of a single threshold can over- or under-estimate the presence of emphysema [11]. Some would also argue that the Perc15/PD15 is a more sensitive and reproducible measure to assess a change in the extent of emphysema in longitudinal and interventional studies (as an aside, we need to agree on a standard nomenclature for these values) [12].

There are important factors that influence the measurement of CT lung density and need standardisation in all studies, particularly in multicentre and longitudinal studies. These include the type and manufacturer of the CT scanner, the radiation dose, slice thickness, the image reconstruction algorithm, body size and the lung volume at which the scan was acquired $[13,14]$. The latter is of particular importance and methods are required to compensate for variations in lung volume, including spirometrically gating the CT scan or using a mathematical approach to correct for lung volume $[15,16]$.

Using these techniques as a surrogate measure to quantify emphysema and taking into account many, but not all of these methodological problems, CT lung density has been used to assess the emphysema

Received: May 122016 | Accepted: May 162016

Conflict of interest: None declared.

Copyright OERS 2016 
phenotype in large-scale cohort studies. This phenotype has been shown to be related to important clinical outcomes, such as the frequency of exacerbations, decline in lung function and mortality [17-20].

The phenotypic characterisation of emphysema is also more complicated, since the pattern (centrilobular, panlobular or pleural-based emphysema) and distribution of emphysema may represent a relevant subtype that is not assessed by these density threshold-based methods. These subtypes can be classified, on visual assessment or by specially developed software, which recognises emphysema patterns [21, 22], and they may have a different genetic background and display different clinical characteristics [22, 23].

CT lung imaging has also been used to quantify airway remodelling. However, the methodology in this case is more problematic [14]. Currently, there are commercially available software packages that enable reconstruction of the airways from volumetric datasets of CT images to create three-dimensional airway trees from the trachea to the fifth- or sixth-generation airways. Unfortunately, there are many different algorithms in use for this purpose and there is no consensus on which software is the most accurate. Some methods, such as the commonly used "full width at half maximum" (FWHM) method, may systematically overestimate airway wall area, especially in smaller airways [24].

Two approaches have been proposed to quantify airway dimensions. The first is to calculate the percentage of the total airway area occupied by its wall (WA\%), an index less dependent on the airway size than wall area [25]. The second approach that was developed to try and alleviate the error in the WA\% caused by measuring smaller airways is the square root of wall area of an idealised airway with an internal perimeter of $10 \mathrm{~mm}$ (Pi10), which is calculated using a linear regression of all measured bronchi to obtain a value characterising an individual patient [26]. A major limitation of these two approaches is that changes may be due to modifications either in the airway lumen, airway wall or both. Further limitations are the relatively low range of values and the overlap with normal findings. There is currently no consensus on the most appropriate dimension to use.

Using these techniques to measure airway dimensions, little or no thickening or narrowing of airway walls is seen in COPD patients [27]. However, WA\% has been shown to correlate with the forced expiratory volume in $1 \mathrm{~s}\left(\mathrm{FEV}_{1}\right)$ and the residual volume/total lung capacity ratio but not the diffusing capacity of the lung for carbon monoxide [28]. Furthermore, studies have shown that airway wall measurements are associated with pathological changes in the airways [29], symptoms of dyspnoea [26] and chronic bronchitis [30], and the frequency of exacerbations [18].

The measurement of airway dimensions by CT scanning is challenging since these measurements are influenced by a number of factors. These include partial volume averaging, the applied reconstruction kernel, the software used to make the measurements, the radiation dose and lung volume [13, 14]. Labelling and categorising bronchi by generation and location are important, and are a further source of variation [31].

A further important limitation is that the small airways $2-3 \mathrm{~mm}$ in diameter that are responsible for the airflow limitation in COPD are below the resolution of the CT scanner. To address this problem, one study showed that the wall thickness in the small airways, measured by histology, was correlated with the wall area in the intermediate-sized airways measured by CT, suggesting that the dimensions of larger airways could be used a surrogate for changes in the small airways [29]. Other investigators have used less direct measurements of airway remodelling in small airways by using CT estimates of gas trapping. This technique applies a density threshold (e.g. $<856 \mathrm{HU})$ to expiratory CT lung images to delineated lung areas that contain trapped gas [32]. Studies have shown that CT measurements of gas trapping correlate well with other measurements of lung function in subjects with COPD [33]. However, one limitation of this technique is that regions of low attenuation may also reflect areas of hyperinflation or emphysema rather than small-airway disease. To discriminate regions of trapped gas from emphysema, an alternative approach has been to co-register expiratory and inspiratory CT images to generate parametric response maps (PRMs), to determine gas trapping from these matched images as emphysematous or due to gas trapping because of small airway disease, termed "functional small-airway disease" [34]. These PRM measurements of gas trapping and emphysema have been shown to be associated with pulmonary function tests, health-related quality of life and frequency of exacerbations [34].

Several studies have used CT imaging to assess both emphysema and large airway dimensions or gas trapping as a surrogate functional measure of small-airway disease. These studies also have distinguished emphysema or airway-dominant and mixed phenotypes within COPD cohorts $[35,36]$ with variable proportions of patients falling into these categories in different COPD populations. These different CT phenotypes may relate to different pulmonary function parameters [37]. Patients with the emphysema-dominant phenotype are associated with low body mass index [38], severe dyspnoea [39], rapid decline of FEV1 [19] and high respiratory-related mortality [40]. Patients with the airway-dominant phenotype are associated with frequent chronic cough [26] and frequent exacerbations [18], although both emphysema and airway wall thickening have been linked to frequent exacerbations [18]. However, a crucial issue in previous studies is the lack of consensus about the upper limits of normal for both the extent of emphysema and the airway wall thickness. 
Furthermore, although emphysema- and airway-dominant phenotypes have been studied extensively, studies of the mixed phenotype are limited but have shown associations with more severe dyspnoea and more frequent hospitalisations than the other CT-based phenotypes [41].

In this issue of the European Respiratory Journal, SUBRAMANIAN et al. [42] report how they applied CT assessment of emphysema and airways to the EvA (Emphysema versus Airway Disease) study cohort, a multicentre project to study mechanisms and identify biomarkers of emphysema and airways disease in COPD. The objective of this study was to delineate imaging-based emphysema-dominant and airway disease-dominant phenotypes using quantitative CT.

The study addressed some, but not all, of the problems described above in assessing emphysema and airway phenotypes in a multicentre study. The scanning protocol was standardised across the 10 participating centres on either of two scanner types. Perc15 and \%WA were measured as the measures of emphysema and airway dimensions, respectively. Particular emphasis was placed on standardising lung density and airway measurements by the use of dedicated emphysema and airway phantoms, which were scanned in all centres and indices were derived to generate centre-specific regression equations using a method that was validated in preclinical phantom studies.

There were clear differences in unstandardised Perc15 and \%WA between different manufacturer and model CT scanners. Preclinical densitometry and airway phantom studies demonstrated that standardisation reduced variability by $79-84 \%$ and, when applied to clinical studies, significantly reduced the variability in measurements due to scanner non-conformity and led to more robust phenotypes.

There are limitations to this study. The measurements of emphysema using the Perc15 was fairly well standardised and the use of phantoms with volumetric adjustment of the values was shown to improve reproducibility of the measurements. However, the measurements of airways were more problematic. Although the use of airway phantoms improved the variability measurements between scanners, the measurements of airways was limited to measurements of the right S1 segmental airway, based on a previous study showing that this measurement was representative of the remodelling in the large and small airways [29], which would now be considered an inferior measure. Furthermore, the metric used to assess changes in the airway wall was the \%WA, which as described earlier, has considerable limitations. Gas trapping as a surrogate for small-airway disease was not assessed, so what was measured was large-airway disease.

Using these techniques, four imaging-derived phenotypes were identified, reflecting "emphysema-dominant", "airway disease-dominant", "mixed" disease and "mild" disease. There was no difference for age, sex or smoking history among the four phenotypes. The majority of patients were in the group classified as having a "mild" COPD phenotype and when the fixed FEV1/forced vital capacity ratio of 0.7 was replaced by the lower limit of normal as a diagnostic criterion for COPD, only $68 \%$ in the "mild" group were still classified as having airflow obstruction, suggesting that almost one-third of subjects in this "mild" group may not have COPD.

The relationships of these phenotypes with clinical parameters and outcome measures was fairly limited in this study, compared with previous similar studies. The emphysema-dominant group had significantly higher lung volumes, lower gas transfer coefficient, lower oxygen and carbon dioxide tensions, higher haemoglobin, and higher blood leukocyte numbers than the airway disease-dominant group, and patients with mild disease had better spirometric measures than the other phenotypes, most of which might have been expected. The group with mixed pathology had upper lobe-predominant emphysema but no clear distinguishing features when compared to the other phenotypes, which does not match the findings from previous studies and may be due to differences in the methodology used to assess emphysema, and particularly airways, between these studies.

What this study demonstrates is there is a need to reach a consensus on the most appropriate method for quantifying emphysema and airways in COPD studies. This has largely been achieved for the measurement of emphysema but not for airways, where there is also a need to undertake longitudinal studies to determine the contributions of CT quantified airways disease for predicting outcomes in patients with COPD.

\section{References}

1 Agusti A, Calverley PM, Celli B, et al. Characterisation of COPD heterogeneity in the ECLIPSE cohort. Respir Res 2010; 11: 122.

2 Han MK, Agusti A, Calverley PM, et al. Chronic obstructive pulmonary disease phenotypes: the future of COPD. Am J Respir Crit Care Med 2010; 182: 598-604.

3 Agusti A, Bel E, Thomas $\mathrm{M}$, et al. Treatable traits: toward precision medicine of chronic airway diseases. Eur Respir J 2016; 47: 410-419.

4 Hayhurst MD, MacNee W, Flenley DC, et al. Diagnosis of pulmonary emphysema by computerised tomography. Lancet 1984; 2: 320-322.

5 Lynch DA. Progress in imaging COPD, 2004-2014. J COPD 2014; 1: 73-82.

6 Han MK. Clinical correlations of computed tomography imaging in chronic obstructive pulmonary disease. Ann Am Thorac Soc 2013; 10: S131-S137. 
7 Gould GA, MacNee W, McLean A, et al. CT measurements of lung density in life can quantitate distal airspace enlargement - an essential defining feature of human emphysema. Am Rev Respir Dis 1988; 137: 380-392.

8 Gevenois PA, De M, De V, et al. Comparison of computed density and macroscopic morphometry in pulmonary emphysema. Am J Respir Crit Care Med 1995; 152: 653-657.

9 Gevenois PA, de Maertelaer V, De Vuyst P, et al. Comparison of computed density and microscopic morphometry in pulmonary emphysema. Am J Respir Crit Care Med 1996; 154: 187-192.

10 Coxson HO, Leipsic J, Parraga G, et al. Using pulmonary imaging to move chronic obstructive pulmonary disease beyond FEV1. Am J Respir Crit Care Med 2014; 190: 135-144.

11 Muller NL, Staples CA, Miller RR, et al. 'Density mask'. An objective method to quantitate emphysema using computed tomography. Chest 1988; 94: 782-787.

12 Stolk J, Dirksen A, Lugt AA, et al. Repeatability of lung density measurements with low-dose computed tomography in subjects with alpha-1-antitrypsin deficiency-associated emphysema. Invest Radiol 2001; 36: 648-651.

13 Coxson HO. Chairman's summary. Proc Am Thorac Soc 2008; 5: 874-877.

14 Mets OM, Jong PA, Ginneken B, et al. Quantitative computed tomography in COPD: possibilities and Limitations. Lung 2012; 190: 133-145.

15 Zagers H, Vrooman HA, Aarts NJ, et al. Assessment of the progression of emphysema by quantitative analysis of spirometrically gated computed tomography images. Invest Radiol 1996; 31: 761-767.

16 Stoel BC, Putter H, Bakker ME, et al. Volume correction in computed tomography densitometry for follow-up studies on pulmonary emphysema. Proc Am Thorac Soc 2008; 5: 919-924.

17 Thomsen LH, Shaker SB, Dirksen A, et al. Correlation between emphysema and lung function in healthy smokers and smokers with COPD. J COPD Foundation 2015; 2: 204-213.

18 Han MK, Kazerooni EA, Lynch DA, et al. Chronic obstructive pulmonary disease exacerbations in the COPD Gene study: associated radiologic phenotypes. Radiology 2011; 261: 274-282.

19 Vestbo J, Edwards LD, Scanlon PD, et al. Changes in forced expiratory volume in 1 second over time in COPD. N Engl J Med 2011; 365: 1184-1192.

20 Celli BR, Locantore N, Yates J, et al. Inflammatory biomarkers improve clinical prediction of mortality in chronic obstructive pulmonary disease. Am J Respir Crit Care Med 2012; 185: 1065-1072.

21 Mishima $\mathrm{M}$, Hirai $\mathrm{T}$, Itoh $\mathrm{H}$, et al. Complexity of terminal airspace geometry assessed by lung computed tomography in normal subjects and patients with chronic obstructive pulmonary disease. Proc Natl Acad Sci USA 1999; 96: 8829-8834.

22 Castaldi PJ, San J, Estépar R, et al. Distinct quantitative computed tomography emphysema patterns are associated with physiology and function in smokers. Am J Respir Crit Care Med 2013; 188: 1083-1090.

23 Smith BM, Austin JH, Newell JD, et al. Pulmonary emphysema subtypes on computed tomography: the MESA COPD study. Am Med 2014; 127: 94.

24 Nakano Y, Whittall KP, Kalloger SE, et al. Development and validation of human airway analysis algorithm using multidetector row CT. Proc SPIE 2002; 4683: 460-469.

25 Nakano Y, Wong JC, Jong PA, et al. The prediction of small airway dimensions using computed tomography. Am J Respir Crit Care Med 2005; 171: 142-146.

26 Grydeland TB, Dirksen A, Coxson HO, et al. Quantitative computed tomography measures of emphysema and airway wall thickness are related to respiratory symptoms. Am J Respir Crit Care Med 2010; 181: 353-359.

27 Smith BM, Hoffman EA, Rabinowitz D, et al. The Multi-Ethnic Study of Atherosclerosis (MESA) COPD Study and the Subpopulations and Intermediate Outcomes in COPD Study (SPIROMICS). Comparison of spatially matched airways reveals thinner airway walls in COPD. Thorax 2014; 69: 987-996.

28 Nakano Y, Muro S, Sakai H, et al. Computed tomographic measurements of airway dimensions and emphysema in smokers. Correlation with lung function. Am J Respir Crit Care Med 2000; 162: 1102-1108.

29 Nakano Y, Wong JC, Jong PA, et al. The prediction of small airway dimensions using computed tomography. Am J Respir Crit Care Med 2005; 171: 142-146.

30 Mair G, Maclay J, Miller JJ, et al. Airway dimensions in COPD: relationships with clinical variables. Respir Med 2010; 104: 1683-1690.

31 Montaudon M, Berger P, Dietrich G, et al. Assessment of airways with three-dimensional quantitative thin-section CT: in vitro and in vivo validation. Radiology 2007; 242: 563-572.

32 Zach JA, Newell JD Jr, Schroeder J, et al. Quantitative computed tomography of the lungs and airways in healthy non-smoking adults. Invest Radiol 2012; 47: 596-602.

33 Hersh CP, Washko GR, Estépar RS, et al. Paired inspiratory-expiratory chest CT scans to assess for small airways disease in COPD. Respir Res 2013; 14: 42.

34 Galbán CJ, Han MK, Boes JL, et al. Computed tomography-based biomarker provides unique signature for diagnosis of COPD phenotypes and disease progression. Nat Med 2012; 18: 1711-1715.

35 Hoesein FA, Schmidt M, Mets OM, et al. Discriminating dominant computed tomography phenotypes in smokers without or with mild COPD. Respir Med 2014; 108: 136-143.

36 Regan EA, Lynch DA, Curran-Everett D, et al. Clinical and radiologic disease in smokers with normal spirometry. JAMA Intern Med 2015; 175: 1539-1549.

37 Sun XW, Gu SY, Li QY, et al. Pulmonary function parameters in high-resolution computed tomography phenotypes of chronic obstructive pulmonary disease. Am J Med Sci 2015; 349: 228-233.

38 Ogawa E, Nakano Y, O'Hara T, et al. Body mass index in male patients with COPD: correlation with low attenuation areas on CT. Thorax 2009; 64: 20-25.

39 Boschetto P, Quintavalle S, Zeni E, et al. Association between markers of emphysema and more severe chronic obstructive pulmonary disease. Thorax 2006; 61: 1037-1042.

40 Haruna A, Muro S, Nakano Y, et al. CT scan findings of emphysema predict mortality in COPD. Chest 2010; 138: 635-640.

41 Van T, Ogawa E, Trang LT, et al. Mixed phenotype of airway wall thickening and emphysema is associated with dyspnea and hospitalization for chronic obstructive pulmonary disease. Ann Am Thorac Soc 2015; 12: 988-996.

42 Subramanian DR, Gupta S, Burggraf D, et al. Emphysema- and airway-dominant COPD phenotypes defined by standardised quantitative computed tomography. Eur Respir J 2016; 48: 92-103. 